\title{
The Principle of Permanent Electrical Storage of Plant Seeds
}

\author{
Zhe Yin \\ Department of Mathematics, Yanbian University, China
}

\begin{abstract}
This paper clarifies the principle of permanent electrical storage of plant seeds.
Key words: Electricity storage; light; gravitational wave
\end{abstract}

\section{Introduction}

It has been proposed in 2010 that substances change to the direction of increase of atomic weight and molecular weight[2] and it has also been proposed that spiral trajectory of gravitational waves [1,3] and the variable force field is the condition of energy conversion in February 2016[7,8]. The motion trajectory of the electrons, proposed the law of motion that, spiral trajectory gravitational wave produces entanglement in the process of convergence and divergence, increases the energy, in the trajectory of Mobius', and spiral gravitational wave convergences inwards in March 2016[3,7,8]. Inward convergence is equivalent that the centripetal force is greater than buoyancy and other external field forces, outward divergence equivalent buoyancy and other external field force is greater than the centripetal force. The following description provides the use of gravitational wave theory, to elucidate the propagation pattern of light between the electrons of the gravitational waves. The light has the entanglement between the periodic and the optical blocks with the same properties. The degree of entanglement between the light blocks is expressed by relative energy. Each photoperiod contains four sub-process morphologies, each sub-process morphology, forming a separate, electron-group and entangled form of relative energy set. This paper presents the principle of permanent electrical storage of plant seeds.

\section{Theoretical preparation}

Theorem 1 (Gravitational waves existence theory) [3,4,5,6,7,8,9,10,13,14].

There are A, B two points. A is the wave source of gravitational field, and B is a point in the gravitational field. The existence of energy rotational motion (including proton, neutron, atomic nucleus or planet) at $\mathrm{A}$ is the necessary and sufficient condition of the existence of gravitational waves at B. Direction is the bidirection of the path tangent at point $\mathrm{B}$, and the limit of the convergence direction is A point.

Theorem 2 (Gravitational wave stability theory) [3,4,5,6,7,8,9,10,13,14].

$\mathrm{A}$ is a gravitational wave source, $\mathrm{B}$ is a point in gravitational field of $\mathrm{A}$. The necessary and sufficient conditions of stable gravitational wave at B point are:

$$
\frac{d r}{d \theta}=b, \text { (b is a constant). }
$$

Inference of theorem 2: If a gravitational wave track meet Archimedean spiral, this must be stable gravitational waves.

There is, $r=a+b \theta$.

$\mathrm{r}$ is the distance of $\mathrm{A}$ to $\mathrm{B}$; $\mathrm{a}$ is the spiral length of $\mathrm{A}$ to $\mathrm{B}$; $\mathrm{b}$ is the distance between the pirals.

\section{The nature of light and the permanent electrical storage principle of plant seeds.}

According to the gravitational wave equation, the periodic law of light is obtained. gravitational waves are the propagation of light. Light is the same form of electronic, ntangled particles in the form of transmission. Photons have relative energy and quality. Through the electronic entanglement, supplement and increase the energy, gradually increase the avelength.

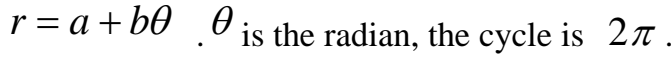

Theorem 1: The wave of light is periodic and its period is $2 \pi$. ?

According to the independence, symmetry, monotonic principle, light has only four forms. Four sub-process morphologies of the photoperiod).

\section{Theorem 2: Four independent morphological equations of light}

The first sub-process morph equation: $r=a+b(2 k \pi+0), k=0,1,2,3, \ldots$.

The second sub-process morph equation: $r=a+b(2 k \pi+\pi / 2), k=0,1,2,3, \ldots$. 
The third sub-process morph equation: $r=a+b(2 k \pi+\pi), k=0,1,2,3, \ldots$.

The fourth sub-process morph equation: $r=a+b(2 k \pi+3 \pi / 2), k=0,1,2,3, \ldots$.

Anywhere on earth, four kinds of photon clusters coexist. The relative energy of the photon is proportional to the wavelength. The four bases of DNA receive energy, and the two deoxynucleotide chains store energy. The energy is used for DNA transcription. When the genes are produced, DNA receives four photon sets of energy. The first photon subset and the fourth photon subset are monotonically increasing in energy. When the DNA metabolic energy is stored, only the first photon and the fourth photon are collected.

The principle of permanent electrical storage of plant seeds: DNA base A and T, G and C accept energy, generate electrical energy, stored in two spiral chains. DNA energy is used for transcription. Among them, DNA acid energy comes from light energy. DNA alkaline energy from the food. DNA base between the hydrogen, plays the role of the valve.

The water on the earth is influenced by the gravitational waves of the sun and the gravitational waves of the earth. Earth gravitational wave length is short, the sun gravitational wave length is long. The gravitational wave of the sun is high in purity and the gravitational wave of the earth is low. Normal water molecules are relatively balanced, requiring independent, without charge.

- The water molecules inside the creature are in a relatively shielded state. Shielding the role of the Earth's gravitational waves. That only accept the role of solar gravitational waves.

- Biological internal water molecules, in the role of solar gravitational waves, divided into positive and negative poles, gel state, charged. Water molecules form an electrical circuit between.

- Plant seeds have endosperm, cotyledons, seed coat and micropyle. Endosperm and cotyledons provide nutrients for seed development. The micropyle is formed by the development of the bead of the ovule. For the seed germination of water when the channel[15].

\section{Theorem 3: The principle of permanent electrical storage of plant seeds}

There are two sources of energy for plant seeds. One is absorbed from the roots of nutrients, stored in the endosperm or cotyledons. Determine the alkalinity or positive charge potential source; the other is the light absorbed from the sunlight, stored in the seed coat. Determine the acidity or negative charge potential source. Can be conditional for permanent preservation. When the water molecules are sucked from the wells and the electrical circuits are formed, a potential difference is generated and discharge is started. This is the energy that the plant DNA gene begins to transcribe.

The seed coat and the peel are connected, and there is also an electronic difference between the fruit and the pulp. The shelf life is the period of resistance to Earth's gravitational waves. Epidermal destruction, there is electronic difference of biological water immediately into ordinary mixed water, easy to oxidation. According to the needs of DAN[11,12], you can use endosperm or cotyledon nutrients, arbitrary generation of various mRNA and ncRNA.

Similarly, the demand for light energy is also determined by genetic DNA. Different plants, the four bases of DNA needs and arrangement is different. Therefore, the genetic DNA on the four photon demand is not the same. This is the reason why the size and color of the flowers of different plants are different. It is important to emphasize that the process of storing electricity and the process of discharge is a reverse process. Seeds in the formation and growth period, the direction of the electron is from outside to inside, the seed germination period, the electrical direction is from the inside out. When the base of DNA receives light energy, the direction of the electrons of the base is $\mathrm{C}$ to $\mathrm{G}, \mathrm{T}$ to $\mathrm{A}$. DNA transcription process, the direction of electrons is $\mathrm{A}$ to $\mathrm{U}, \mathrm{G}$ to $\mathrm{C}$, and $\mathrm{G}$ to $\mathrm{U}$.

\section{Conclusion}

In this paper, the principle of gravitational wave is used to clarify the principle of permanent electrical storage of plant seeds. On the relevant research, will play a catalytic role.

\section{References}

[1]. Weber, J., 1959, 2016. Gravitational Wave, Laser Interferometer Gravitational Wave Observatory. 2016.2.11.23, 30.

[2]. Zhe yin, 2010. The co-planarity and symmetry principle of earthquake occurrence. Int. J. Geosci. 1(1), 38-43.

[3]. Zhe yin, 2016. Zhe Yin's energy and wave theorem. Int. J. Engg. Adv. Res. Technol. 2(3), 44-45.

[4]. Zhe yin, 2017a. The function and relation of proton and neutron. Int. J. Recent Sci. Res. 8(1), 15321-15324.

[5]. Zhe yin, 2017b. Effect of Gravitational Wave on DNA Base. Int. J. Recent Sci. Res. 8(2), 15705-15707.

[6]. Zhe yin, 2017c. The formation of elements. IOSR J. Appl. Phys. 9(2), 08-13.

[7]. Zhe yin, Liu, D., 2016a. Electron trajectory. Int. J. Appl. Sci. Math. 3( 2), 56-57.

[8]. Zhe yin, Liu, D., 2016b. Causes of sound wave on the moon. Int. J. Eng. Appl. Sci. 3(2), 56-58. 
[9]. Zhe yin, Zhang, W., Bie, H., 2016. Zhe Yin's theorem of molecular biology. Acad. J. Sci. Res. 4(4), 99-102.

[10]. Zhe yin, 2017d. The periodicity of light and the nature of chlorophyll. J. Curr. Res. Biosci. Plant Biol. 4(4), 46-48.

[11]. Zhe yin,yunfei Guo,2014. Application of the ZheYin's Gene Inherits Law, Open Journal of Genetics,Vol.4,No.6,pp:434-438.

[12]. Zhe yin,2012. The Law of Genetic Inheritance, Open Journal of Genetics,Vol.2, No.1,pp: 47-50.

[13]. Zhe yin,2017e. EFFECTS OF GRAVITATIONAL WAVE ON GENETIC INHERITANCE, European Journal of Basic and Applied Sciences, Vol. 4 No. 1, pp:35-40.

[14]. Zhe yin,2017f.Visible Light Formation Process, Journal of Applied Physics (IOSR-JAP), Volume 9, Issue 2, PP 37-39.

[15]. Zhe yin,yunfei guo,2015.Bio-segregation research to understand the biological metabolism, Pure Appl. Biol., 4(2): 259-260. 